\title{
CASE REPORT OF ADULT-ONSET CHARCOT MARIE TOOTH TYPE X
}

\author{
R Suherlim $^{1}$, A.A.A. Putri Laksmidewi ${ }^{2}$, and NKA Sudiarini ${ }^{3}$ \\ Correspondence: putri_laksmidewi@unud.ac.id \\ ${ }^{1}$ Department of Neurology, Neurology Resident, Faculty of Medicine, Udayana University, Sanglah Hospital, Denpasar, Bali, Indonesia \\ ${ }^{2}$ Department of Neurology, Faculty of Medicine, Udayana University, Sanglah Hospital, Denpasar, Bali, Indonesia
} ${ }^{3}$ Department of Neurology, Tabanan Public Hospital, Bali, Indonesia

\section{Article History:}

Received: June 6, 2021

Accepted: November 15, 2021

Published: January 1, 2022

\section{Cite this as:}

Suherlim R, Laksmidewi AAAP, Sudiarini NKA. Case report of adult-onset charcot marie tooth type x. Malang Neurology Journal; 2022.8:68-71. DOI: http://dx.doi.org/10.21776/ub.mnj .2022.008.01.14

\section{ABSTRACT}

Charcot-Marie-Tooth (CMT) or Hereditary Motor and Sensory Neuropathy (HMSN) is the most common hereditary peripheral nerve disease with progressive chronic weakness, muscle atrophy, and sensory disturbances. There are several types and subtypes of CMT with their respective clinical manifestations. In this article, we reported a patient with of CMT type X. A 43-year-old male patient was referred to a neurology clinic with weakness in both limbs for 2 years, accompanied by tingling and sensory disturbance in both hands and feet. There are several of his family members who had similar complaints. Lumbosacral magnetic resonance imaging (MRI) examination revealed mild nucleus pulposus herniation. Electroneuromyography (ENMG) examination revealed demyelinating sensory motor polyneuropathy. Histopathological examination of nerve biopsy showed demyelination of the sural nerve. It is hard to make a diagnosis of CMT, because it requires high suspicion from clinicians once encounter a suspected case and also need to supported by sophisticated equipment such as electrophysiological examinations, nerve biopsy examinations, and genetic examinations. It is vital for clinicians for being able to diagnose CMT correctly and provide treatment as soon as possible in order to maintain the patients' quality of life.

Keywords: Charcot-Marie-Tooth, adult onset, type X

\section{Introduction}

Charcot-Marie-Tooth (CMT) is the most common group of hereditary diseases that cause peripheral nerve damage characterized by chronic progressive weakness, muscle atrophy, and sensory disturbances. ${ }^{1,2}$ There are several types of CMT, namely CMT1, CMT2, CMT3, CMT4, and CMT X. Each type has its own characteristics. ${ }^{3,4}$

Patients with CMT initially experience weakness in the lower leg muscles causing difficulty lifting the leg (foot drop) and high stepped gait accompanied by frequent tripping or falling. Foot deformities such as high arches or hammertoes are also common with CMT. As the disease progresses, weakness and atrophy begin to develop in the muscles of the upper limbs (thenar and hypothenar). Charcot-Marie Tooth also causes degeneration of myelin sheath or axons of sensory nerves, which cause disturbances to feel pain, temperature, touch, vibrations, and propioception. ${ }^{4}$ Recent studies have even shown that in type X, CMT can cause impaired cognitive function. ${ }^{5}$

In diagnosing CMT, while taking history aside from signs and symptoms, it is important to know a detailed family history to look for inheritance patterns of this disease. ${ }^{4}$ Charcot-Marie Tooth has been known for a long time, but unfortunately there is no definitive therapy to cure it up until now. The number of clinical trials has not yielded satisfactory results. ${ }^{6,7}$

\section{Case Report}

A 43-year-old male patient was referred to a neurology clinic with weakness in both limbs for 2 years, which was worsening in the last 1 year. Initially, the patient was still able to walk well, but in the last 1 year, it was said that walking was difficult and making the patient unable to work. The patient also complained about tingling sensation and sensory disturbance in both hands and legs which appeared simultaneously with walking difficulty. Tingling in the hands is felt from the wrists to the fingers, tingling in the legs is felt from the knees to the toes. Defecate and urinate are normal. A history of falling or hitting the neck or waist is refuted. Prior to this illness, the patient had no history of systemic disease.

On physical examination, patient was compos mentis (GCS E4V5M6) and hemodynamically stable, no cranial nerve palsy, grade 4+ spastic paraparesis, bilateral ankle clonus, bilateral babinksi reflex, and lower limb muscle atrophy.

Based on the examination, the patient was initially diagnosed with suspected space occupying lesion (SOL) of spinal cord. The patient was hospitalized for observation and further examination. The patient underwent a lumbosacral MRI without contrast which resulted in a herniated nucleus pulposus (HNP) at the L4-L5 and L5-S1 levels which slightly pressed both roots, but no cerebrospinal liquor flow (CSF) obstruction was seen on MR myelography (Figure 1). These results did not support 


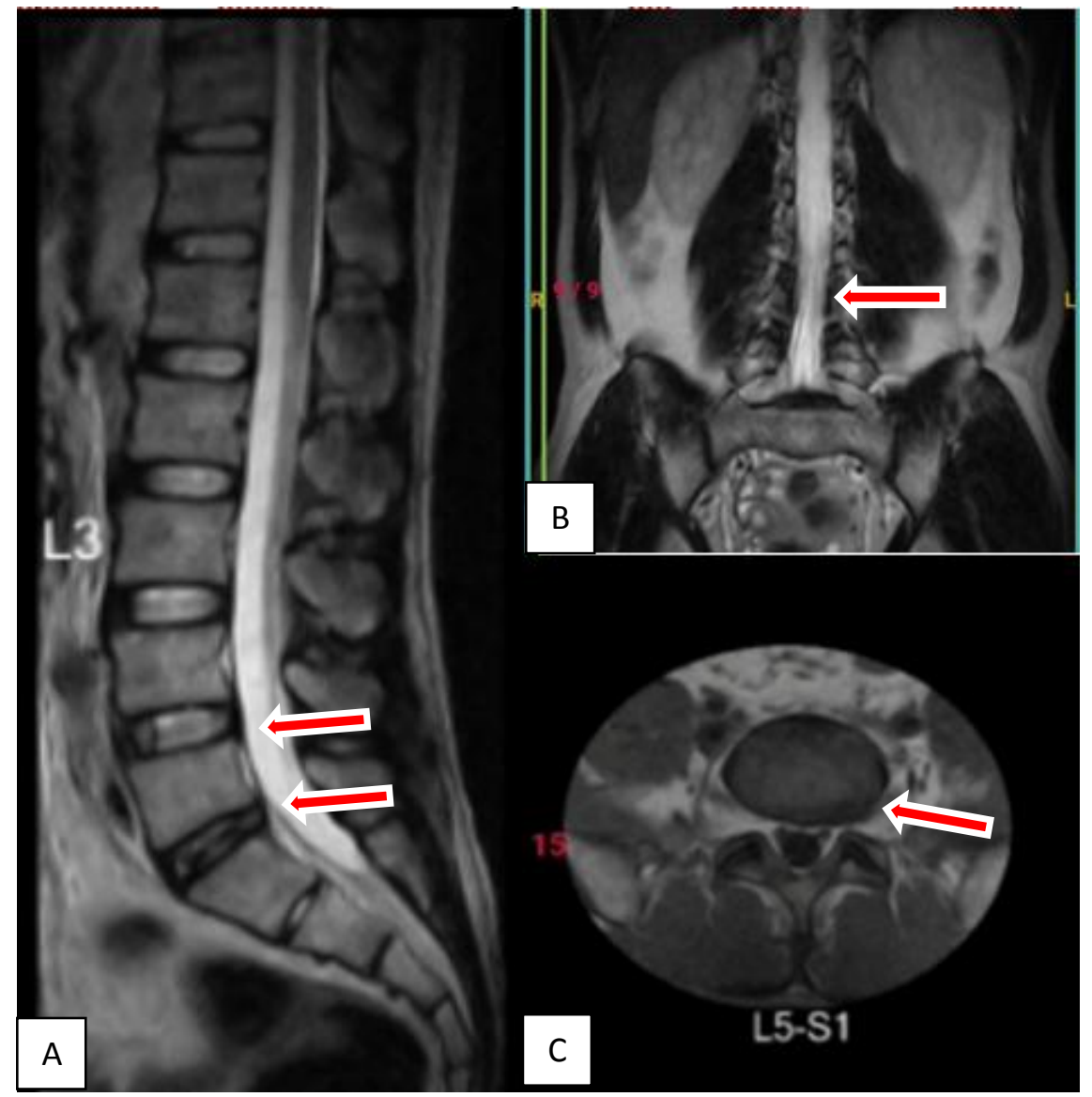

Figure 1. Axial, sagittal, and coronal sections of lumbosacral MRI without contrast. There's disc bulging on the level of L4-5 and L5-S1 that caused mild compression on central canal dan nerve roots (arrow).

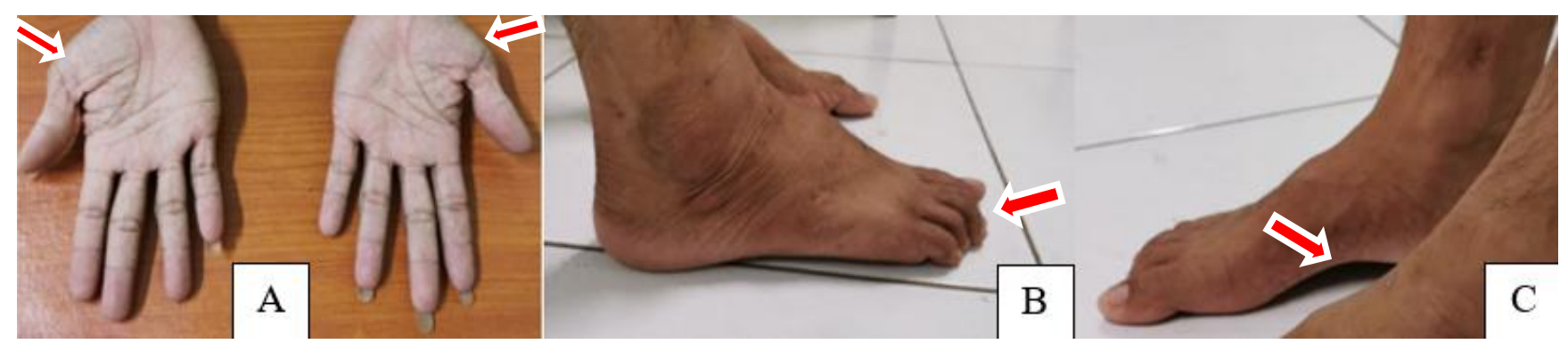

Figure 2. (A) Bilateral thenar muscle hypotrophy (B) Hammer toes (C) Pes Clavus

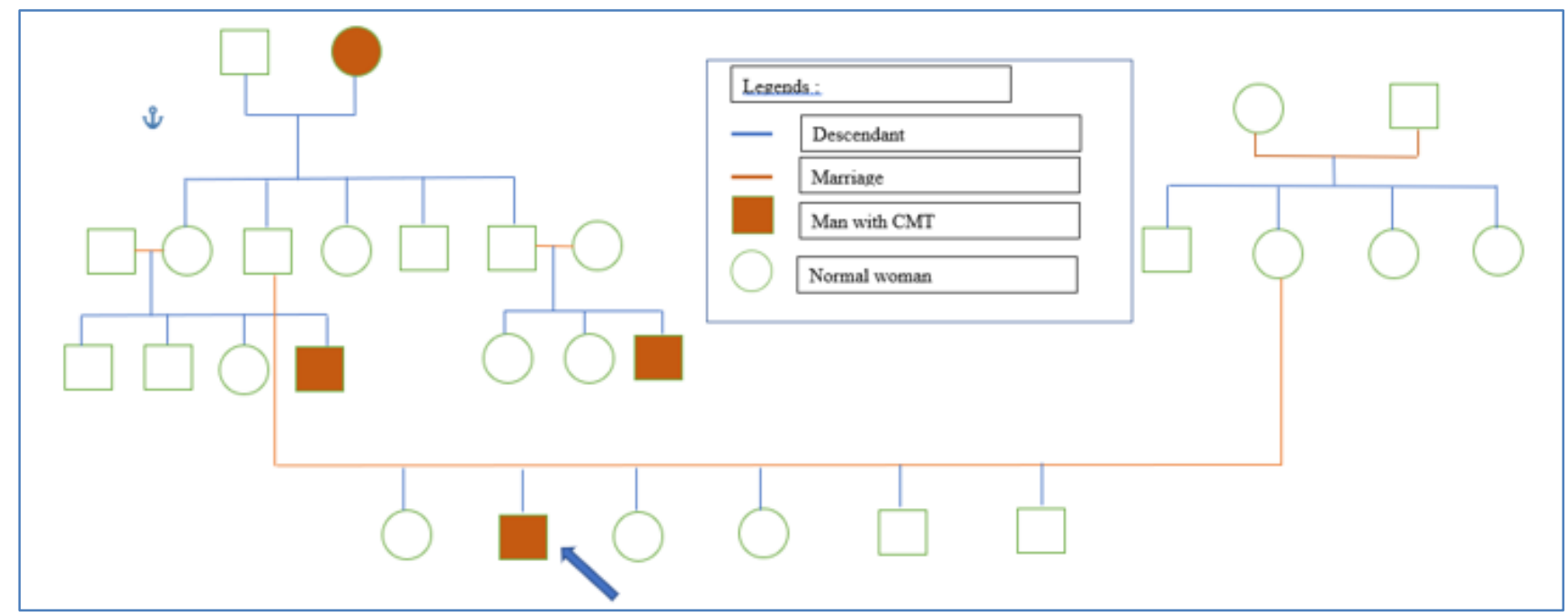

Figure 3. Family tree of the patient. There are 3 family members from father's side with similar symptoms as the patient. 


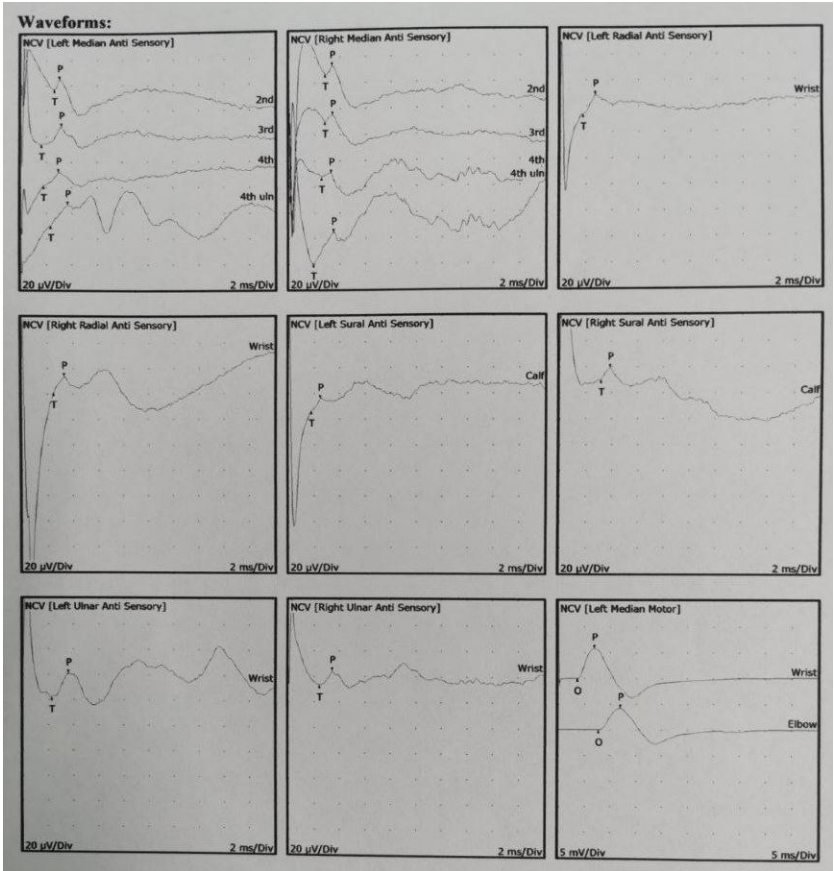

Figure 4. ENMG examination . CMAP: Prolonged distal latency of left radial nerve, bilateral peroneal nerve, and bilateral tibial nerve. Low amplitude of right peroneal nerve and bilateral tibial nerve. SNAP: Prolonged distal latency of right radial nerve. Low amplitude of bilateral median nerve.

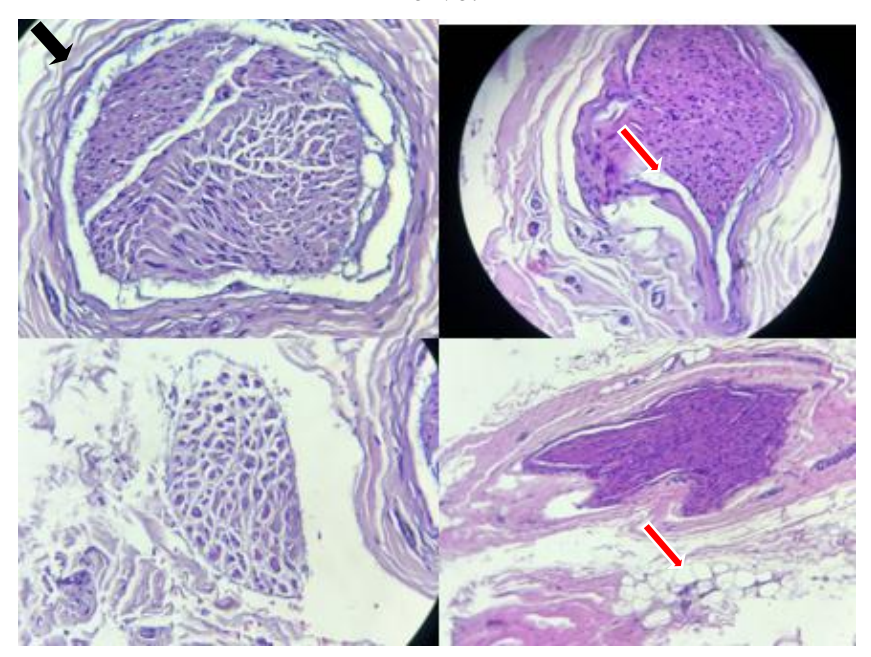

Figure 5. Pathology anatomical of sural nerve biopsy . Onion bulb appearance (black arrow) and lipid degeneration (red arrow)

the severity of the patient's symptoms, so that the patient was re-evaluated and then an ENMG was planned. Due to facility limitations, the patient was discharged and scheduled for ENMG examination in the neurology clinic.

The patient said that there are several members of his family who also experienced weakness of the limbs and chronic tingling, but none of them had symptoms as severe as that of the patient. (Figure 3) The patient was diagnosed with suspected hereditary motor and sensory neuropathy / Charcot-Marie-Tooth disease.

The ENMG concluded that the patient had demyelinating sensory motor polyneuropathy. (Figure 4) Furthermore, to confirm the diagnosis, a nerve biopsy examination was done. The results of the sural nerve biopsy showed that demyelination and fibrosis, outside the large nerve fibers there was a union bulb. (Figure 5) The morphological features are suitable for the demyelination of the sural nerve in CMT.

The patient was diagnosed with CMT and given vitamin C, mecobalamin, and amitriptyline. The patient wanted to continue his treatment in a health facility close to his home because CMT is a chronic disease and treatment will take a long time.

\section{Discussion}

Charcot-Marie-Tooth (CMT) is the most common hereditary neuropathy disease in the world with 1-3 cases per 100,000 population. There are no CMT epidemiology data in Indonesia. ${ }^{8}$ In this article, the patient was a 43 years old male who had weakness in both limbs and tingling sensation in all four extremities.

The clinical manifestation of CMT is a combination of hereditary motor and sensory deficits, either autosomal dominant, autosomal recessive, or via the $\mathrm{X}$ chromosome. The onset of symptoms varies depending on the type. The common manifestations of CMT are consistent with the description of lower motor neuron (LMN) type motor deficits. Common sensory manifestations are glove-andstocking-type hypesthesia or paresthesia. These symptoms will gradually worsen, becomes more and more disturbing over time. ${ }^{1,9}$

In classic CMT, the motor neurologic deficit that occurs should be the LMN type because of damage to the peripheral nerves. However, characteristics of upper motor neuron (UMN) lesions were found in this patient such as increased leg tone, increased deep tendon reflexes, and the presence of clonus. There is a case report of similar case in which CMT patient was reported with clinical pyramidal tract lesions. A 16 years old male patient with bilateral leg hypertonus, increased bilateral tendon and superficial reflexes, cavo varus leg deformities, hypotrophy of intrinsic muscles of the hand, peroneal muscle, and extensor digitorum brevis muscle. There is also grade 4 weakness in the distal muscles of all four extremities. ENMG results showed motor sensory polyneuropathy in all four extremities. After genetic evaluation, the patient was diagnosed with CMT type 1D with the mutation in the ERG2 gene. $^{10}$

History taking, physical examination, and advance investigations in this reported case support the diagnosis of CMT type X. Epidemiologically, CMTX is the second most common type of CMT after CMT1. ${ }^{4}$ Male CMTX patients have more severe symptoms than female patients due to the abnormalities in X chromosome. In women, it is more often a heterogeneous case of inheriting one of the $\mathrm{X}$ defective genes from a parent. CMTX has a dominant X-linked inheritance pattern. Based on that, there is no possible male-to-male transmission in the family history. ${ }^{11,12}$

Charcot-Marie-Tooth type X accounts for about $10 \%$ of the overall prevalence of CMT. The results of CMTX electrophysiology do not meet the criteria of CMT1 and CMT2 so that they are classified into intermediate CMT, namely no neurological hypertrophy, nerve conduction velocity of $38-45 \mathrm{~m} / \mathrm{s}$, prolonged distal motor latency. ${ }^{11,12}$

The histopathological examination of sural nerve biopsy with hematoxylin eosin staining showed a demyelination, 
onion bulb appearance, and fatty degeneration. Demyelination occurs when schwann cell dysfunction occurs (eg HMSN) or myelin sheath damage (eg GuillainBarre syndrome). Repeated processes of demyelination and remyelination are associated with schwann cell proliferation. If this process occurs excessively, it will form concentric layer of cytoplasm and Schwann cells basement membrane surrounding thin myelinated axons that form multiple layers surrounding the axon like an onion so it is called an onion bulb appearance. These can progress to axonal lesions. ${ }^{13}$

In this case, the diagnosis of CMT cannot be confirmed with certainty because there is no genetic examination. However, based on existing data, the diagnosis of CMT type $\mathrm{X}$ is very possible. It is necessary to check the patient's family members to get a more definite family history. The patient must be monitored constantly because CMT cannot be cured, but his quality of life must be maintained.

\section{Conclusion}

It is hard to diagnose Charcot-Marie-Tooth disease. Charcot-Marie-Tooth also has many types and variants, each of which has its own distinctive symptoms. Therefore, it is vital for clinicians for being able to diagnose CMT correctly and provide treatment as soon as possible in order to maintain the patients' quality of life.

\section{Conflict of Interest}

The authors have nothing to disclose.

\section{Acknowledgement}

The authors would like to thank the patient, patient's family, staffs and residents of neurology in Udayana University for their support in our efforts making this report published.

\section{References}

1. Timothy CP. Charcot-Marie-Tooth and other hereditary motor and sensory neuropathies; 2018. [Cited 2021 Mar 10]. Available from: https://emedicine.medscape.com/article/1173104overview

2. Thomas DB. Charcot-Marie-Tooth (CMT) hereditary neuropathy overview; 2021. [Cited 2021 Mar 10]. Available from:
https://www.ncbi.nlm.nih.gov/books/NBK1358/

3. Lokesh RM, Raju K, and Nagarathna PKM. A detailed study on a rare disease: Charcot Marie Tooth (CMT). Journal of Pharmacy and Biological Sciences; 2014; 9(2): 29-39.

4. National Institute of Neurological Disorders and Stroke. Charcot-Marie-Tooth disease fact sheet. [Updated 2020; Cited 2021 Mar 10]. Available from: https://www.ninds.nih.gov/Disorders/PatientCaregiver-Education/Fact-Sheets/Charcot-Marie-

Tooth-Disease-Fact-Sheet\#3092_3

5. Dimitrios K, et al. Evidence for cognitive deficits in $\mathrm{X}-$ linked Charcot-Marie-Tooth disease. Journal of the International Neurophysiological Society; 2020: 1-9. DOI: https://doi.org/10.1017/S1355617719001188

6. Donald MC, Evan MP, and Nicholas EJ. Management of Charcot-Marie-Tooth disease: Improving long-term care with a multidisciplinary approach. Journal of Multidisciplinary Healthcare; 2016; 9: 7-19. DOI: 10.2147/JMDH.S69979

7. Laurie $G$ and Michael S. Update on Charcot-MarieTooth disease. Current Opinion Neurology; 2015; 28 (5): 462-7. DOI: 10.1097/WCO.0000000000000237

8. Lidiane CLSB, et al. Epidemiologic study of CharcotMarie-Tooth disease: A systematic review. Neuroepidemiology; 2016; 46: 157-65. DOI: $10.1159 / 000443706$

9. Kenny M, Jimmy S, Octaviani. Advanced onset Marie Tooth Charcot Disease. Neurona; 2020; 31 (2): 1-7. Avalaible from: http://www.neurona.web.id/paper/1152.pdf

10. Carlo F, et al. Charcot-Marie-Toot disease with pyramidal features due to a mutation of EGR2 gene. Acta Biomedica Journal; 2019; 90 (1): 104-6. DOI: 10.23750/abm.v90i1.6951.

11. Berciano J, et al. Intermediate Charcot-Marie-Tooth disease: An electrophysiological reappraisal and systematic review. Journal of Neurology; 2017: 1-25. DOI: $10.1007 / \mathrm{s} 00415-017-8474-3$

12. Jonathan M, Anirudh G, and Hoyle JC. CharcotMarie-Tooth: From molecules to therapy. International Journal of Molecular Sciences; 2019; 20: 1-15. DOI: 10.3390/ijms20143419

13. Kumar V, et al. Robbins \& Cotran pathologic basis of disease-8th ed. Philadelphia: Saunders Elsevier. 2010. ISBN: 9781416031215 